Document downloaded from:

http://hdl.handle.net/10251/146714

This paper must be cited as:

Hasiow, B.; Minicka, J.; Zarzynska, A.; Budzynska, D.; Elena Fito, SF. (2018). Defective RNA particles derived from Tomato black ring virus genome interfere with the replication of parental virus. Virus Research. 250:87-94. https://doi.org/10.1016/j.virusres.2018.04.010

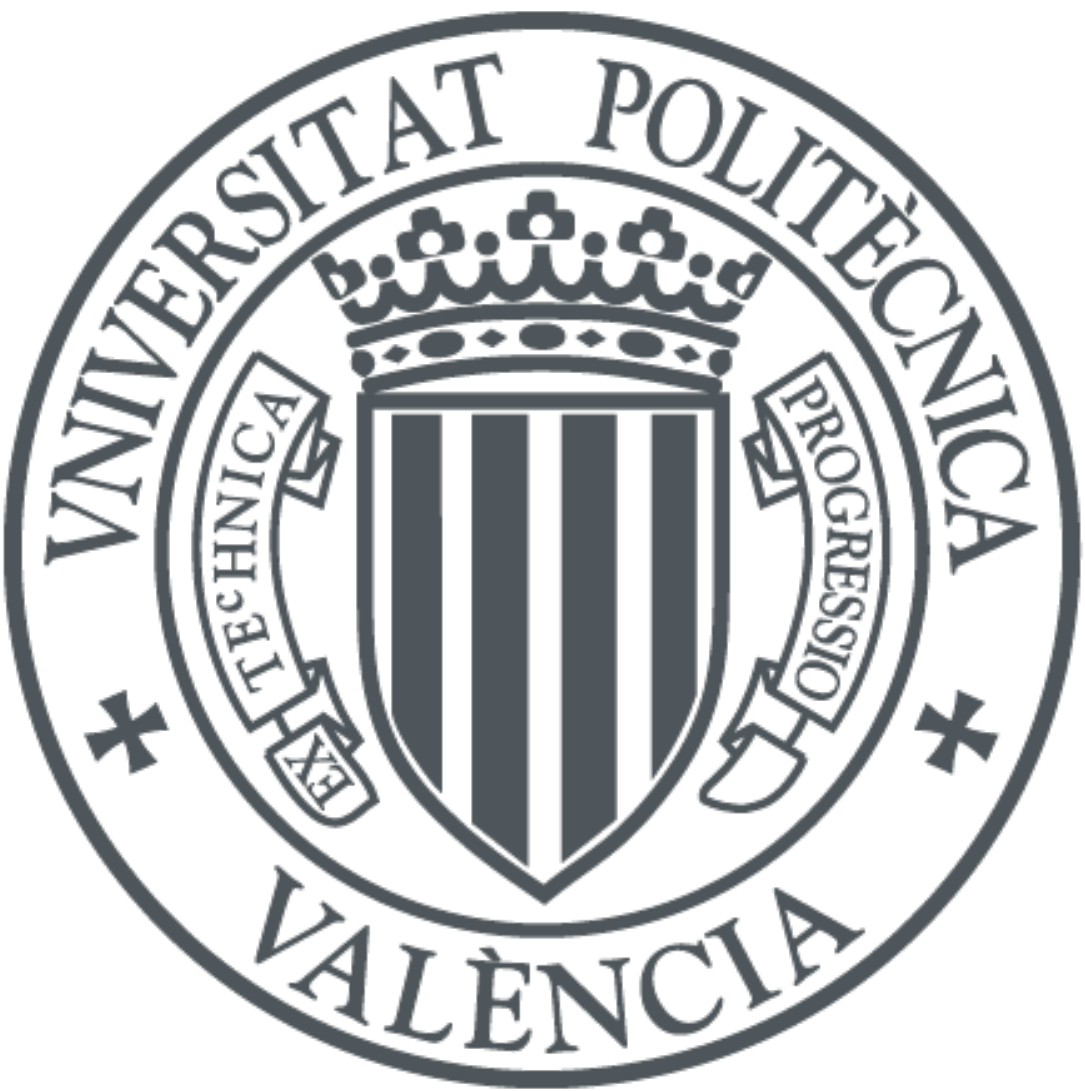

The final publication is available at

https://doi.org/10.1016/j.virusres.2018.04.010

Copyright Elsevier

Additional Information 
See discussions, stats, and author profiles for this publication at: https://www.researchgate.net/publication/324523527

\section{Defective RNA particles derived from Tomato black ring virus genome interfere with the replication of parental virus}

Article in Virus Research · April 2018

DOI: $10.1016 /$ j.virusres.2018.04.010

CITATION

1

5 authors, including:

Beata Hasiów-Jaroszewska

Institute of Plant Protection - National Research Institute

126 PUBLICATIONS 497 CITATIONS

SEE PROFILE

Aleksandra Zarzyńska-Nowak

Institute of Plant Protection - National Research Institute

21 PUBLICATIONS 36 CITATIONS

SEE PROFILE

Some of the authors of this publication are also working on these related projects:

Virus Evolution View project

Project Tempo and Mode View project
READS

139

Julia Minicka

Institute of Plant Protection - National Research Institute

14 PUBLICATIONS 93 CITATIONS

SEE PROFILE

Santiago F Elena

Spanish National Research Council

485 PUBLICATIONS 11,334 CITATIONS

SEE PROFILE 


\section{Accepted Manuscript}

Title: Defective RNA particles derived from tomato black ring virus genome interfere with the replication of parental virus

Authors: Beata Hasiów-Jaroszewska, Julia Minicka, Aleksandra Zarzyńska-Nowak, Daria Budzyńska, Santiago F. Elena

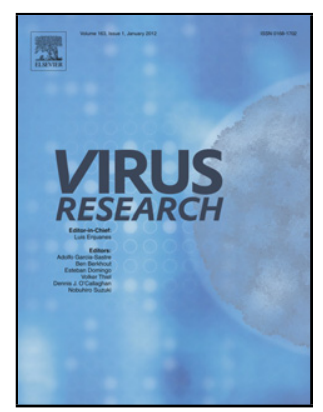

PII: S0168-1702(18)30095-9

DOI: https://doi.org/10.1016/j.virusres.2018.04.010

Reference: VIRUS 97383

To appear in: $\quad$ Virus Research

Received date: $\quad$ 12-2-2018

Revised date: $\quad$ 5-4-2018

Accepted date: $\quad$ 10-4-2018

Please cite this article as: Hasiów-Jaroszewska B, Minicka J, Zarzyńska-Nowak A, Budzyńska D, Elena SF, Defective RNA particles derived from tomato black ring virus genome interfere with the replication of parental virus, Virus Research (2010), https://doi.org/10.1016/j.virusres.2018.04.010

This is a PDF file of an unedited manuscript that has been accepted for publication. As a service to our customers we are providing this early version of the manuscript. The manuscript will undergo copyediting, typesetting, and review of the resulting proof before it is published in its final form. Please note that during the production process errors may be discovered which could affect the content, and all legal disclaimers that apply to the journal pertain. 


\title{
Defective RNA particles derived from tomato black ring virus genome interfere with the replication of parental virus
}

Beata Hasiów-Jaroszewska ${ }^{1}$, Julia Minicka ${ }^{1}$, Aleksandra Zarzyńska-Nowak ${ }^{1}$, Daria Budzyńska $^{1}$, Santiago F. Elena ${ }^{2,3,4}$

${ }^{1}$ Institute of Plant Protection-National Research Institute, Department of Virology and Bacteriology, ul. Wł. Węgorka 20, 60-318 Poznań, Poland

${ }^{2}$ Instituto de Biología Molecular y Celular de Plantas, Consejo Superior de Investigaciones Científicas-Universitat Politècnica de València, València, Spain. ${ }^{3}$ Instituto de Biología Integrativa de Sistemas, Consejo Superior de Investigaciones Científicas-Universitat de València, València, Spain. ${ }^{4}$ The Santa Fe Institute, Santa Fe, New Mexico, USA.

*Correspondence: Beata Hasiów-Jaroszewska: beatahasiow@o2.pl

\section{Highlights}

- Structure and origin of D RNAs associated with two new TBRV isolates were analyzed

- D RNAs of TBRV interfere with parental virus replication

- The interference effect depends on the interplay between TBRV isolate and the host

\begin{abstract}
Tomato black ring virus (TBRV) is the only member of the Nepovirus genus that is known to form defective RNA particles (D RNAs) during replication. Here, de novo generation of D RNAs was observed during prolonged passages of TBRV isolates originated from Solanum lycopersicum and Lactuca sativa in Chenopodium quinoa plants. D RNAs of about $500 \mathrm{nt}$ derived by a single deletion in the RNA1 molecule and contained a portion of the 5, untranslated region and viral replicase, and almost the entire 3' non-coding region. Short regions of sequence complementarity were found at the 5' and 3' junction borders, which can facilitate formation of the D RNAs. Moreover, in this study we analyzed the effects of D RNAs on TBRV replication and symptoms development of infected plants. C. quinoa, S. lycopersicum, Nicotiana tabacum, and L. sativa were infected with the original TBRV isolates
\end{abstract}


(TBRV-D RNA) and those containing additional D RNA particles (TBRV+D RNA). The viral accumulation in particular hosts was measured up to 28 days post inoculation by RTqPCR. Statistical analyses revealed that D RNAs interfere with TBRV replication and thus should be referred to as defective interfering particles. The magnitude of the interference effect depends on the interplay between TBRV isolate and host species.

Keywords: defective RNAs; TBRV; virus accumulation; RT-qPCR; interference

\section{Introduction}

Tomato black ring virus (TBRV) is the type member of Nepovirus genus within the family Secoviridae. Taking into account the differences in the length of RNA2, the degree of sequence identity and serological properties, nepoviruses were classified into three groups: A, B and C (Steinkellner et al., 1992; Sanfaçon, 2009). TBRV belongs to subgroup B and infects a wide range of economically important plants worldwide. Since 1957, there have been reports of significant damage caused by TBRV infection to several important hosts other than tomato: strawberry, potato, celery, and artichoke (Hollings, 1965; Gallitelli et al., 2004). TBRV is transmitted mechanically with plant sap, soil-inhabiting nematodes of Longidorus genus and through the seeds of some plant species (Harrison et al., 1961; Lister and Murant, 1964). The viral genome consists of two single-stranded RNAs of about $7400 \mathrm{nt}$ and $4600 \mathrm{nt}$ in length, respectively, carrying a small covalently attached VPg protein at 5' ends and a poly(A) tail at their 3' ends. Each genomic RNA encodes for a polyprotein, which are proteolytically cleaved into mature functional proteins by the RNA1-encoded protease. RNA1 is responsible for viral replication and polyprotein processing, while RNA2 encodes genes necessary for encapsidation and movement in plants (Mayo and Robinson, 1996; Jończyk et al., 2004).

TBRV infection can be accompanied by subviral particles such as satellite RNAs (satRNAs) and defective RNAs (D RNAs). SatRNAs share little sequence similarity with the viral genomic RNAs, whereas D RNAs are derived from the viral genomic RNAs via a copy choice mechanism resulting in sequence deletion(s). These RNAs are called "defective" since they do not encode any proteins, and their replication, encapsidation and spread depend on the parental (helper) virus (Holland, 1991). D RNAs are synthesized by the viral RNA-dependent RNA polymerase (RdRp), which also replicates the parental virus genomes. D RNAs are often generated de novo during serial passages of viral isolates in one host (Perrault, 1981; Li 
et al., 1989; Hasiów-Jaroszewska et al., 2012). Most of D RNAs contain parts of the noncoding regions of their helper virus' genome and preserve a portion of open reading frame(s) (ORFs) (Romero et al., 1993; Graves et al., 1996; Hernández et al., 1996; Qiu and Scholthof, 2001; Pathak and Nagy, 2009). RNA recombination plays a major role in the production of D RNA particles. The errors made by the RdRp, including template switching, are likely the main mechanism of D RNAs formation. Additionally, highly active "hotspot regions" for RNA recombination, which might promote the formation of D RNAs, very often consist of AU-rich stretches (Kim and Kao, 2001). Pausing of the viral RdRp may be also mediated by the sequence and/or secondary structure of the donor or nascent RNA (Nagy and Simon, 1997). D RNAs can modulate helper virus accumulation, symptoms observed in infected plants and even virus evolution (Holland et al., 1987; Li et al., 1989; Romero et al., 1993; Pathak and Nagy, 2009). D RNAs, which are referred to interfere with the multiplication of their helper viruses, are called "defective interfering RNAs" (DI RNAs).

De novo generation of D RNAs was previously demonstrated during serial passages of chosen TBRV isolates from black locust, black elder, tomato, and zucchini. D RNAs of about 400 - $500 \mathrm{nt}$ in length derived from TBRV RNA1 were classified into two types according to their molecular structure. The first type containing a part of the 5' untranslated region (UTR), segment located in the 5' region of the ORF and a portion of the 3' UTR of RNA1. The second type consisted of a short fragment of the 5' UTR, a portion of the C-terminal end of the RdRp and the almost entire 3' UTR of RNA1. A single deletion, which resulted in the formation of defective particles, covered the region corresponding to the area between 371 $7243 \mathrm{nt}$ or 147 - $6840 \mathrm{nt}$ of RNA1, respectively (nt positions refer to GenBank accession AY157993.1) (Hasiów-Jaroszewska et al., 2012; Rymelska et al., 2013). The role that D RNAs may have in the replication of TBRV is not fully understood. It was observed that the presence of D RNA affects the attenuation of symptoms on infected plants, suggesting its interference with helper virus multiplication (Hasiów-Jaroszewska et al., 2012; Rymelska et al., 2013; Hasiów-Jaroszewska et al., 2016).

In this work, we report the molecular characterization of de novo generated D RNAs of two new TBRV isolates originated from tomato (Solanum lycopersicum L. cv. Moneymaker; Solanaceae family) and lettuce (Lactuca sativa L.; Asteraceae family). Moreover, we analyze the impact of D RNAs on the replication of the helper virus, and the symptoms induced on the infected plants, showing that these D RNAs are, indeed, DI RNAs. 


\section{Materials and methods}

\subsection{TBRV isolates, plant inoculations and growing conditions}

Two TBRV isolates were used to perform the experiments. The first, designated as TBRV-Pi was obtained from a greenhouse tomato, while the second (TBRV-S1) was isolated from a lettuce. The isolates were transferred by mechanical inoculation and maintained in Chenopodium quinoa Willd. (Amaranthaceae family). Plants were grown under greenhouse conditions at a temperature of $22-23^{\circ} \mathrm{C}$ and a photoperiod of $16 \mathrm{~h}$. Purified virus preparations were obtained in sucrose gradient as described previously (Rymelska et al., 2013). Viral RNAs were extracted by a phenol-chloroform procedure (Green and Sambrook, 2012) and the RNAs profile was analyzed by agarose gel electrophoresis. The concentration of each RNA sample was measured in a ND-1000 spectrophotometer (Thermo Fisher Scientific, Wilmington DE, USA) and adjusted to $1 \mu \mathrm{g} / \mu \mathrm{l}$.

\subsection{Virus passages}

The long-term passing of TBRV-Pi and TBRV-S1 was performed in C. quinoa. Viral RNAs at a final concentration of ca. $1 \mu \mathrm{g} / \mu \mathrm{l}$ were inoculated onto Carborundum-dusted leaves of $C$. quinoa in the approximate amount of $3.3 \mu 1$ per leaf. Infected plants were maintained under greenhouse conditions for 7 days post-inoculation (dpi). The initial virus population derived from infected plants was transferred to virus-free plants of the same species and passaged 15 times over a total period of 105 - 110 days. After each passage of $7 \mathrm{dpi}$, the whole plants were collected, their tissues were ground in $2 \mathrm{ml}$ of phosphate buffer (0.05 M, pH 7.2), and the resulting sap was used for mechanically inoculating new plants. After 15 passages, purified viral preparations were obtained, and RNA was isolated using the phenol-chloroform procedure. The RNA profile was analyzed in $1.5 \%$ agarose gel.

\subsection{Amplification, cloning and sequencing of short RNAs}

All molecules shorter than TBRV-genomic RNAs were extracted from the gel and purified using Zymoclean ${ }^{\mathrm{TM}}$ Gel RNA Recovery Kit (ZymoResearch, Irvine CA, USA), according to the manufacturer's instructions. Small RNAs were amplified using Transcriptor One-Step RTPCR Kit (Roche, Mannheim, Germany), according to the manufacturer's instructions. In order to amplify short RNAs, a set of the primers complementary to the conserved 5' and 3' UTRs of both TBRV RNA1 and RNA2 was used (Hasiów-Jaroszewska et al., 2012; Rymelska et al., 
2013). The RT-PCR products were purified using a NucleoSpin Gel and PCR Clean-up Kit (Macherey-Nagel, Düren, Germany) and cloned into a TOPO ${ }^{\mathrm{TM}}$ TA Cloning ${ }^{\mathrm{TM}}$ Kit for Sequencing (Thermo Fisher Scientific, Waltham MA, USA). The recombinant plasmids were transformed into One Shot ${ }^{\mathrm{TM}}$ TOP10 Chemically Competent Escherichia coli (Thermo Fisher Scientific, Waltham MA, USA), according to manufacturer's instructions. Plasmid DNA was

purified using Invisorb ${ }^{\circledR}$ Spin Plasmid Mini Two (Stratec Molecular, Berlin, Germany) and the presence of an insert verified by digestion with appropriate restriction enzymes. At least three independent plasmid DNAs were sequenced using M13F and M13R primers in an external company (Genomed, Warsaw, Poland). In order to determine the extent to which D RNAs correspond to their 5' and 3' fragments of parental genomic RNAs, 5' and 3' RACE Systems for Rapid Amplification of cDNA Ends (Invitrogen, Carlsbad CA, USA) and the following primers: 5'RACE1 5'-CAAAATCGTCAAGGACGATATC-3' and 3'RACE1 5'GTGAAACCTATGCTGCC-3' were used according to the manufacturer's instructions. Furthermore, the cDNA ends were sequenced and compared with the sequences of defective RNAs. A recombination analysis was performed using RDP4 (Martin et al., 2015) and Recco softwares (Maydt and Lengauer, 2016).

\subsection{In vitro transcription of D RNA molecules}

Gel-purified D RNAs served as templates in RT-PCR reaction with primers DF 5'AAAAGAGCTCTAATACGACTCACTATAGCAAATCCTGTAACCAATCAG-3' promoter is underlined) and DR 5'-AAGTCGACAATCTTTTTGTGTCCAACA-3'. The RTPCR reaction was performed using a Transcriptor One-Step RT-PCR Kit (Roche, Mannheim, Germany) in $50 \mu \mathrm{l}$ reaction volume containing $1 \mu \mathrm{l}$ of RNA, $10 \mu \mathrm{l}$ of $5 \times$ reaction buffer, 0.4 $\mu \mathrm{M}$ of each primer, $1 \mu \mathrm{l}$ Transcriptor Enzyme Mix, and $34 \mu \mathrm{l}$ of water. Reverse transcription was performed at $50{ }^{\circ} \mathrm{C}$ for $30 \mathrm{~min}$. The initial denaturation was performed at $94{ }^{\circ} \mathrm{C}$ for 10 min, followed by 35 cycles of denaturation at $94{ }^{\circ} \mathrm{C}$ for $10 \mathrm{~s}$, annealing at $60{ }^{\circ} \mathrm{C}$ for $30 \mathrm{~s}$ and extension at $68{ }^{\circ} \mathrm{C}$ for $1 \mathrm{~min}$. A final extension at $68{ }^{\circ} \mathrm{C}$ for $5 \mathrm{~min}$ was also performed. The RT-PCR products were separated on $1 \%$ agarose gel stained with Midori Green (NIPPON Genetics, Düren, Germany). The obtained RT-PCR products were purified using NucleoSpin Gel and PCR Clean-up (Macherey Nagel, Düren, Germany) and subsequently used for transcription. Transcription was performed using mMESSAGE mMACHINE Kit (Ambion, Austin TX, USA) according to manufacturer's instructions. 


\subsection{Impact of D RNAs on symptoms development and accumulation of the viral RNA}

To determine the impact of D RNAs on symptoms development the RNA isolated from original TBRV isolates, which did not posses any additional RNA particles (TBRV-Pi-D RNA and TBRV-S1-D RNA) was mixed in different combinations (1:1, 1:2 and 2:1 ratios) with RNA transcripts corresponding to D RNAs. The concentration of each RNA sample was measured in a ND-1000 spectrophotometer (Thermo Fisher Scientific, Wilmington DE, USA).

The RNA mixtures (at a final concentration of about $1 \mu \mathrm{g} / \mu \mathrm{l}$ ) were inoculated onto Carborundum-dusted leaves of C. quinoa and Nicotiana tabacum L. cv. Xanthi plants, $10 \mu 1$ per plant. Three plants for each variant were used and maintained under greenhouse conditions $\left(22-23{ }^{\circ} \mathrm{C}, 16 \mathrm{~h}\right.$ photoperiod). The plants infected with TBRV-Pi-D RNA and TBRV-S1-D RNA served as positive controls. All plants were monitored for symptoms development during 28 dpi. The presence of TBRV RNA and D RNAs in infected plants was confirmed by RT-PCR as described above.

Real-time quantitative RT-PCR (RT-qPCR) experiments were performed to determine whether the presence of D RNAs has an impact on the parental virus accumulation. The original TBRV isolates (TBRV-Pi-D RNA and TBRV-S1-D RNA), as well as those obtained after the serial passages that contained D RNAs (TBRV-Pi+D RNA and TBRV-S1+D RNA) were used in these experiments. The quality and concentration of TBRV genomic RNAs and D RNAs was estimated using capillary electrophoresis using Qsep-100 DNA Analyzer (BiOptic Inc., Taipei, Taiwan). The final concentration of genomic RNAs was established of ca. $1 \mu \mathrm{g} / \mu \mathrm{l}$ and used to inoculate the following test plants: S. lycopersicum, C. quinoa, $N$. tabacum, and L. sativa. The RNA accumulation level was analyzed for each studied variant separately for five biological replicates. Each biological replicate was repeated thrice (technical replicates). The experiments were repeated twice.

Ten $\mu$ l of RNA was used for mechanical inoculation of each plant (approximately 3.3 $\mu l$ per each Carborundum-dusted leaf). The plants were maintained for $28 \mathrm{dpi}$ and the symptoms were monitored. The entire experiment was conducted under greenhouse conditions ( $22-23{ }^{\circ} \mathrm{C}, 16 \mathrm{~h}$ photoperiod, $50 \%$ humidity) in closed, monitored compartments. In parallel, mock-inoculated plants (treated only with inoculation buffer) were grown as negative controls. The apical part of the plants infected with the particular variant (TBRVPi+D RNA, TBRV-Pi-D RNA, TBRV-S1+D RNA, and TBRV-S1-D RNA) were taken individually, and total RNAs were extracted using the RNeasy Plant Mini Kit (Qiagen, Hilde, 
Germany) according to the manufacturer's protocol. The RNAs were isolated 7, 14, 21, and 28 dpi, measured with a ND-1000 spectrophotometer (Thermo Fisher Scientific, Waltham MA, USA) and diluted to the final concentration of ca. $10 \mathrm{ng} / \mu \mathrm{l}$. To prepare a standard curve, RNA transcripts of $C P$ gene of both isolates (TBRV-Pi and TBRV-S1) were obtained. First, each cDNAs of the isolates were synthesized using $1 \mu \mathrm{l}$ of appropriate RNAs at a final concentration of $1 \mu \mathrm{g} / \mu 1$, Oligo (dT) primer (200 $\mathrm{nM}$ ) and Transcriptor High Fidelity cDNA Synthesis Kit (Sigma-Aldrich, Darmstadt, Germany), according to the manufacturer's protocol. Then, the $C P$ was amplified using TBRVCP1F 5'ATGAATTCTAATACGACTCACTATAGGCAGACGGGGATTTTGCCTTGG-3' promoter is underlined)

and

TBRVCP1R

5'GCGGCCGCTGCGGGAATAGTTAGAGGACCTGC-3' primers, at an annealing temperature of $60{ }^{\circ} \mathrm{C}$. The resulting PCR products were separated on a $1 \%$ agarose gel to verify the appropriate size of the obtained products and purified using NucleoSpin ${ }^{\circledR} \mathrm{Gel}$ and PCR Clean-up (Macherey-Nagel, Düren, Germany), according to the manufacturer's protocol. RNA transcripts were produced using the mMESSAGE mMACHINE Kit (Ambion, Austin TX, USA) according to the manufacturer's protocol, measured and diluted to the final concentration of $1 \mu \mathrm{g} / \mu \mathrm{l}$, which corresponded to $1.183 \cdot 10^{12}$ copies of viral genomes. Then, the RNA transcripts obtained from both isolates underwent 10-fold serial dilutions from $1.183 \cdot 10^{12}$ to $1.183 \cdot 10^{6}$ copies of viral genomes, using appropriate RNAs from healthy plants as diluents. RT-qPCR reaction was performed using iTaq SYBR Green (Biorad, Hercules CA, USA), CPqF: 5'-CGAAGGCAACTCTGCAA-3' and CPqR: 5'TCCAGTTTCCATGGTTTCTG-3' primers. Each sample was analyzed in three technical replicates. The number of viral genomes in each sample at 7, 14, 21, and 28 dpi was calculated by comparing the obtained results to the values from the standard curve with LightCycler $^{\circledR} 96$ SW 1.1 software (Roche, Mannheim, Germany).

\subsection{Statistical analysis}

Let $n(t)$ be the number of viral genomes quantified by RT-qPCR in a sample $t$ dpi. Then, we can define a new variable $N(t)$ as the cumulative number of genomes produced until time $t$ dpi as $N(t)=\sum_{i=7}^{t} n(i)$. Cumulative number of genomes data were then fitted to a fully factorial statistical model by generalized linear model (GLM) methods in which TBRV isolate (Pi and $\mathrm{Sl}$ ), presence or absence of D RNAs and host species (C. quinoa, L. sativa, N. tabacum, and $S$. lycopersicum) were all treated as orthogonal factors. Time (dpi) was treated as a covariable. 
The model assumed normally distributed errors and a link function (choice based in the lowest BIC among alternative models tested). The significance of each factor, the covariable and all of their interactions was evaluated by means of likelihood-ratio tests $(L R T)$ that asymptotically follow a $\chi^{2}$ probability distribution. A factor may be significant but still have very little effect in explaining the observations. Therefore, it is important to quantify the contribution of each factor to the predictive power of a model. To achieve this, the magnitude of the effects associated to each factor were evaluated using two different statistics, the $\eta_{P}^{2}$ statistic that represents the proportion of total variability attributable to a given factor when controlling for the other effects $\left(\eta_{P}^{2}<0.05\right.$ are considered as small and $\eta_{P}^{2} \geq 0.15$ as large), and the percentage of total variance explained by each of the factors. All statistical analyses were performed with SPSS version 23 software (IBM, Armonk NY, USA).

\section{Results}

\subsection{Spontaneous generation of TBRV D RNAs}

The RNA analysis of TBRV-Pi and TBRV-S1 isolates originated from tomato and lettuce, respectively revealed the presence of two typical TBRV RNAs: RNA1 (7400 nt) and RNA2 (4600 nt). No additional bands on the agarose gel were observed (Fig. 1). After 15 serial passages in $C$. quinoa, additional small RNAs of about $500 \mathrm{nt}$ were observed for both TBRV isolates (Fig. 1). Small RNAs were amplified using primer pair P128/P2 (HasiówJaroszewska et al., 2012; Rymelska et al., 2013), cloned and sequenced. The sequences of particular D RNAs obtained from three independent clones were identical. Sequences of D RNAs associated with TBRV-Pi and TBRV-S1 were deposited in GenBank under accession numbers MG458222 and MG458223, respectively. Sequence comparison with the parental genome sequences confirmed their defective character.

\subsection{Structure and origin of D RNAs}

The recombination analysis revealed that both D RNAs derived from the TBRV RNA1 as a result of single deletion between 147 - $6862 \mathrm{nt}$ (positions refer to TBRV-Pi accession number MG458220). De novo formed D RNAs of TBRV-S1 and TBRV-Pi shared the characteristic of the previously-described second type of TBRV D RNAs: a small fragment of 5' UTR (20 nt), a portion of the C-terminal end of $R d R p$ and the almost entire 3' UTR (Fig. 2A). Based on their structure, both D RNA particles were classified to the second type of TBRV D RNAs. D 
RNAs of TBRV-S1 and TBRV-Pi were very similar to each other and shared $99.1 \%$ sequence identity.

Analysis of the nucleotide sequences near junction sites suggest potential mechanisms which may operate to generate TBRV D RNAs. The sequence motif AGAAAAG within the RdRp coding region of TBRV-S1 and TBRV-Pi is a perfect complementary inverted repeat of the 5' UTR sequence CUUUUCU (Fig. 2 B), suggesting the formation of a hairpin structure between these two sequences. Such hairpin structure may also play a potential role during replication and recombination by pausing of the polymerase following by re-initiation of RNA synthesis and as a consequence, in D RNAs formation.

\subsection{Impact of D RNAs on symptoms development}

Significant differences in symptoms development were observed between plants infected with the TBRV+D RNA versus those infected with TBRV-D RNA. The presence of D RNAs decreases the symptoms severity in all TBRV/D RNAs combinations however the attenuation of symptoms was especially observed when a 1:2 ratio of TBRV/D RNAs was inoculated. Generally, the plants infected with TBRV-Pi-D RNA displayed more severe symptoms. On C. quinoa, chlorosis, leaf malformation and growth reduction were observed. On N. tabacum, chlorotic ringspots and mosaic were noticed. On S. lycopersicum, severe necrosis was notified (Fig. 3A, C, E). Plants of L. sativa were reduced in growth and leaf mosaic was observed (Fig. 3G). When D RNAs were present, the symptoms produced by TBRV appeared one or two days later than normal. Plants infected with TBRV+D RNA were characterized by milder symptoms, single chlorotic or necrotic spots, slight ringspots and mosaics (Fig. 3B, D, F, H). Similar effect was observed for TBRV-S1 (data not shown), which suggests that D RNAs may interfere with the replication of the parental helper virus. The comparison between TBRV-Pi-D RNA and TBRV-S1-D RNA revealed an interesting biological difference between the isolates; TBRV-S1-D RNA systematically produced less severe symptoms on tomato. This finding is in agreement with our previous results showing that TBRV isolates originated from tomato cause severe necrosis on this host whereas isolates originated from other host species cause symptomless infection or single necrotic spots on tomato (Rymelska et al., 2013).

\subsection{Virus quantification and statistical analyses}


A comparison was made of the accumulation level of the two TBRV isolates in presence and absence of the D RNAs and in different plant species by RT-qPCR. Data are summarized in Fig. 4, while Table 1 shows the results of the statistical analyses described in section 2.6. The statistical model fitted to the data contains three main factors and one covariable, their six pairwise interactions, four three-way interactions, and one four-way interaction. Such parameter-rich model requires of a careful analysis. We will describe the results shown in Table 1 starting from the second row of main effects and moving down to increasing number of interactions. The first noticeable result is that, overall, TBRV-Sl accumulates $\sim 8 \%$ more than TBRV-Pi, regardless the other factors. Despite being significant, the contribution of this factor to the total observed variability is rather small $\left(\eta_{P}^{2}<0.05\right.$ and no contribution to explaining the observed variance). Next, the presence of D RNA reduces the accumulation of TBRV in $\sim 26 \%$, on average. The contribution to this factor to the predictive power of the model can be considered as moderate according to the $\eta_{P}^{2}$ value and the $3.12 \%$ of variance explained. Next line in Table 1 assess the overall effect of host species in TBRV accumulation. This factor explains, by far, most of the observed variability in TBRV accumulation (both $\eta_{P}^{2}$ and the percentage of variance explained suggest is a factor of large effect). On average, TBRV accumulates to the highest observed level into C. quinoa, $~ 38 \%$ less in N. tabacum, $\sim 77 \%$ less in L. sativa, and down to $\sim 91 \%$ less in S. lycopersicum. The fifth line in Table 1 simply tells that accumulation increases with time in a highly significant manner. This effect can be easily appreciated in Fig. 4 (notice the log-scale) for all curves.

The next four lines in Table 1 evaluate the effect of pairwise interactions between the three factors and the covariable. Notice that TBRV isolate-by-presence/absence of D RNAs and TBRV isolate-by-time are not included in the table because they were not significant. The TBRV isolate-by-host species is highly significant, with moderate to large effect $\left(\eta_{P}^{2}\right.$ is close to 0.15 and the percentage of variance explained is $3.21 \%$ ), indicating that each one of the TRBV isolates accumulates to different levels on different hosts. For both TRBV-Pi and TRBV-Sl the maximum accumulation is observed in $C$. quinoa (black symbols in Fig. 4) and the lowest in S. lycopersicum (blue symbols in Fig. 4). However, for TBRV-Pi, accumulation in L. sativa is similar to that observed in S. lycopersicum $\left(\sim 4 \cdot 10^{9}\right.$ genomes) whereas TBRV-Sl accumulation in L. sativa is five time higher and closer to the values observed in N. tabacum and $C$. quinoa $\left(\sim 2 \cdot 10^{10}\right.$ genomes). Next line in Table 1 evaluates the contribution of the presence/absence of D RNAs-by-host to accumulation, in other words whether the observed negative effect of D RNAs on TBRV accumulation (see previous paragraph) depends on the 
host species. The effect is statistically significant $(P=0.009$, with strong power $>75 \%)$, though its contribution is small $\left(\eta_{P}^{2}<0.05\right.$ and with a very small percentage of explained variance). Next line in Table 1 tests whether the negative effect associated to the presence of D RNAs in TBRV accumulation is homogeneous with time. In other words, whether the slope of the growth curves in presence or absence of D RNAs is equal in both cases. A significant effect has been detected, indicating differences in slope. However, the effect is rather small in magnitude $\left(\eta_{P}^{2}<0.05\right.$ and very small contribution to the total variance). This small yet significant effect can be easily understood looking back to Fig. 4. Comparing lines and symbols of equal colors, it is obvious that the presence of D RNAs in all cases shifts down the accumulation curves, although curves remain parallel, with the exception TBRV-Pi in $L$. sativa, where the presence of D RNAs dramatically changes the slope of the accumulation curve. This case drives the significance of this interaction. Finally, the ninth line in Table 1 evaluates whether differences exist in the slopes of accumulation curves among hosts. A highly significant effect has been observed, of large effect $\left(\eta_{P}^{2}>0.15\right.$ and up to $10.68 \%$ of variance explained by it). Again, this is obvious in Fig. 4: comparing different curves of different color, some are flatter than others, meaning that TRBV accumulates at different rates in different hosts.

Next, we will discuss the three-way interactions. Out of the four possible cases, only two are significant. The interaction TRBV isolate-by-presence/absence of D RNAs-by-host species is perhaps the most interesting one as it provides information about the nature of this biological system. The interaction is significant $(P=0.002)$ and with strong power $(>75 \%)$, indicating it is a robust conclusion. However, the magnitude of the effect is small $\left(\eta_{P}^{2}<0.05\right.$ and less than $1 \%$ of contribution to explain the observed variation). Nonetheless, this interaction indicates that the negative effect of the presence of D RNAs in the accumulation of the different TRBV isolates indeed depends on the species of the infected host. This can be seen in Fig. 4. The very large effect of the presence of D RNAs in the accumulation of TBRV-Pi in L. sativa (green lines and symbols) can be compared with the very small effect on TBRV-Sl in the same host. These differences can be collated with the situation in $N$. tabacum (red lines and symbols), in which the effect of the D RNAs is smaller for both TBRV isolates but the shape of the curves is different. These two cases can be also compared with the situation in S. lycopersicum (blue lines and symbols), in which the negative effect of adding D RNAs is slightly larger for TBRV-Sl than for TBRV-Pi. The last line in Table 1 tests whether the previously discussed interaction TBRV isolate-by-host changes along time. 
Indeed, the effect is also highly significant though of small magnitude $\left(\eta_{P}^{2}<0.05\right.$ and only $1.39 \%$ of contribution to explain the observed variance).

In conclusion, rigorous statistical analyses support the notion that the presence of D RNAs interfere with TBRV accumulation, and that this interference depends both on the viral isolate being considered and on the host species being infected.

\section{Discussion}

Defective RNA particles are deletion/and/or rearrangement variants of the viral genomes created during replication. Most of D RNAs contain parts of non-coding regions of their helper virus' genome and preserved a portion of ORF(s) (Romero et al., 1993; Graves et al., 1996; Qiu and Scholthof, 2001; Pathak and Nagy, 2009). D RNAs are often generated de novo as a result of prolonged passages in one host (Perrault, 1981; Li et al., 1989; Holland, 1991; Pogany et al., 1995; Hasiów-Jaroszewska et al., 2012). It has been also shown that both host and environmental factors strongly affected the size and formation of the de novo generated D RNAs (Llamas et al., 2004). Most of them suppress virus accumulation and attenuate symptoms in virus-infected plant. D RNA particles, which are reported to interfere with multiplication of their helper viruses, are called "defective interfering RNAs" (DI RNAs). The phenomenon of D RNA interference with the replication of helper virus has been previously reported for many plant viruses, including Carmovirus, Potexvirus, Bromovirus, Tospovirus, and Cucumovirus (Graves et al., 1996). D RNAs have been also found to be associated with TBRV representing Nepovirus genus (Jończyk et al., 2004; HasiówJaroszewska et al., 2012; Rymelska et al., 2013).

In this study, we confirmed that TBRV D RNAs might be generated spontaneously ( $d e$ novo) from TBRV genome during serial of passages in one host. The observed D RNAs of TBRV-Pi and TBRV-S1 isolates resemble the previously characterized TBRV D RNAs of second type, since they derived by internal deletion in the RNA1 molecule and preserved a small portion of the C-terminal end of the $R d R p$ gene. This type of D RNAs have been previously described for TBRV isolates from zucchini, black elder and tomato (HasiówJaroszewska et al., 2012; Rymelska et al., 2013). It suggests that D RNAs structure is rather correlated with the presence of particular motifs in helper virus genome promoting their 
formation than with the host. On the other hand, D RNAs of second type are being prevalent in TBRV population as only two D RNAs associated with the isolates from black locust were classified as a first type. The preservation of the $R d R p$ open reading frame suggest its role in the accumulation of D RNAs in plants. D RNAs formation might be promoted by the presence of highly active hotspot regions for RNA recombination, which consist of AU-rich stretches or a stable secondary structure. Analysis of the sequences flanking the recombination sites in TBRV RNA1 revealed the presence of a U-rich regions and short complementary sequences which may lead to the formation of heteroduplex. Such hairpin structure may also play a potential role during replication and recombination by the pausing of the polymerase following by re-initiation of RNA synthesis and, as a consequence, in D RNA formation (Pathak and Nagy, 2009). All TBRV D RNAs described to date derived exclusively from the RNA1 component which suggests that this particular sequence complementarity and/or secondary structures formed between complementary regions can facilitate D RNA formation. The other possibility is that D RNAs derived only from RNA1 because the RNA polymerase-encoding RNA1 component has some preferences during viral replication.

As of now, the significance of D RNAs in a TBRV replication cycle remains unknown. However, it was proposed that D RNA may play a role in symptom attenuation and virus accumulation (Hasiów-Jaroszewska et al., 2012; Hasiów-Jaroszewska et al., 2016). The results from the studies here reported showed that D RNAs was a factor significantly affecting development of symptoms and virus accumulation in infected plants. The presence of D RNAs resulted in decreasing of disease severity and a reduction in parental virus accumulation in all tested plant species and thus they should be properly referred to as DI RNAs. The presence of DI RNAs has a major effect on symptom attenuation which is not directly related to a reduction of viral RNA accumulation levels. Slight differences between particular hosts and isolates were observed, which suggests that DI RNA formation and its implication for viral replication is triggered by many host factors. Three major mechanisms of interference by DI RNAs have been described: (i) competition for viral and host resources, which impairs virus replication and attenuates the symptoms; (ii) DI RNAs-triggered posttranscriptional gene silencing (PTGS gene-silencing response); and (iii) modulation of the functions of viral factors (Holland et al., 1987; Szittya et al., 2002; Pathak and Nagy, 2009; Lukhovitskaya et al., 2013). Our studies also revealed that the dynamics of TBRV accumulation were different for each host, that they were affected in different manner by the 
presence of DI RNAs and that this effect is host-dependent. The biologically active TBRV cDNA clone have been recently obtained (Zarzyńska-Nowak et al., 2017) which allows us to do further studies of the interference phenomenon of TBRV DI RNAs.

DI RNAs are useful tools for analyzing the processes and mechanisms of recombination, studying viral replication and discovering host factors which affect this phenomenon (Pathak and Nagy, 2009). DI RNA sequence-based constructs are also frequently used for gene silencing and the expression of heterologous proteins. DI RNA particles represent a major controlling element of virus replication. The understanding of mechanism involved in the D RNA formation, knowledge about interaction and competition between DI RNAs and the helper virus is a step toward new, innovative strategies to protect plant against viruses.

\section{Funding information}

This work was supported by the National Science Centre, Poland [grant number 2017/25/B/NZ9/01715]; Ministry of Science and Higher Education, Poland [grant number IP2014 014973] (to B.H.-J.) and Spain Agencia Estatal de Investigación-FEDER [grant number BFU2015-65037P] (to S.F.E.). The funding bodies were not involved into the design of the study, analysis, and interpretation of data in the manuscript.

\section{References}

1. Gallitelli, D., Rana, G.L., Volvas, C., Martelli, G.P., 2004. Viruses of globe artichoke: An overview. J. Plant Pathol. 86, 267-281.

2. Graves, M.V., Pogany, J., Romero, J., 1996. Defective interfering RNAs and defective viruses associated with multipartite RNA viruses of plants. Semin. Virol. 7, 399-408.

3. Green, M.R., Sambrook, J., 2012. Molecular cloning: A laboratory manual. Cold Spring Harbor Laboratory Press, New York.

4. Harrison, B.D., Mowat, W.P., Taylor, C.E., 1961. Transmission of a strain of Tomato black ring virus by Longidorus elongatus (Nematoda). Virology 14, 480-485.

5. Hasiów-Jaroszewska, B., Borodynko, N., Figlerowicz, M., Pospieszny, H., 2012. Two types of defective RNAs arising from the Tomato black ring virus genome. Arch. Virol. $157,569-572$. 
6. Hasiów-Jaroszewska, B., Minicka, J., Rymelska, N., Zarzyńska-Nowak, A., 2016. Defective RNAs derived from the genome of Tomato black ring virus interfere with its replication. $13^{\text {th }}$ International Plant Virus Epidemiology Symposium, p. 92.

7. Hernández, C., Carette, J.E., Brown, D.J.F., Bil, J., 1996. Serial passage of Tobacco rattle virus under different selection conditions results in deletion of structural and nonstructural genes in RNA2. J. Virol. 70, 4933-4940.

8. Holland, J.J., 1991. Defective viral genomes, in: Fields, B.N., Knipe D.M. (Eds.), Virology $2^{\text {nd }}$ ed. Raven Press, New York, pp. 151-165.

9. Holland, J.J., Semler, B.L., Jones, C., Perrault, J., Reid, L., Roux, L., 1987. Role of DI, virus mutation, and host response in persistent infections by envelope RNA viruses. Symp. Mol. Cell. Biol. 11, 57-73.

10. Hollings, M., 1965. Some properties of celery yellow vein, a virus serologically related to Tomato black ring virus. Ann. Appl. Biol. 55, 459-470.

11. Jończyk, M., Borodynko, N., Figlerowicz, M., Pospieszny, H., 2004. Small defective interfering RNAs arising from Tomato black ring virus (TBRV) genome. FEBS J. P2.201.

12. Jończyk, M., Le Gall, O., Palucha, A., Borodynko, N., Pospieszny, H., 2004. Cloning and sequencing of full-length cDNAs of RNA1 and RNA2 of a Tomato black ring virus isolate from Poland. Arch. Virol. 149, 79-807.

13. Kim, M.J., Kao, C., 2001. Factors regulating template switch in vitro by viral RNAdependent RNA polymerases: implications for RNA-RNA recombination. Proc. Natl. Acad. Sci. USA 98, 4972-4977.

14. Li, X.H., Heaton, L.A., Morris, J.T., Simon, A.E., 1989. Turnip crinkle virus defective interfering RNAs intensify viral symptoms and are generated de novo. Proc. Natl. Acad. Sci. USA 86, 9173-9177.

15. Lister, R.M., Murant, A.F., 1964. Seed-transmission of nematode-borne viruses. Ann. Appl. Biol. 59, 49-62.

16. Llamas, S., Sandoval, C., Babin, M., Pogany, J., Bujarski J.J., Romero J., 2004. Effect of the host and temperature on the formation of defective RNAs associated with Broad bean mottle virus. Phytopathology 94, 69-74.

17. Lukhovitskaya, N.I., Thaduri, S., Garushyants, S.K., Torrance, L., Savenkov, E.I., 2013. Deciphering the mechanism of defective interfering RNA (DI RNA) biogenesis reveals 
that a viral protein and the DI RNA act antagonistically in virus infection. J. Virol. 87, 6091-6103.

18. Martin, D.P., Murrell, B., Golden, M., Khoosal, A., Muhire, B., 2015. RDP4: Detection and analysis of recombination patterns in virus genomes. Virus Evol. 1, vev003.

19. Maydt, J., Lengauer, T., 2016. Recco: Recombination analysis using cost optimization. Bioinformatics 22, 1064-1071.

20. Mayo M., Robinson D., 1996. Nepoviruses: molecular biology and replication, in: Harrison, B.D., Murant, A.F. (Eds.), The Plant Viruses. Springer Science, New York, pp. $139-185$.

21. Nagy, P.D., Simon, A.E., 1997. New insights into the mechanism of RNA recombination. Virology 235, 1-9.

22. Pathak, K.N., Nagy, P.D., 2009. Defective interfering RNAs: foes or viruses and friends of virologists. Viruses 1, 859-919.

23. Perrault J. Origin and replication of defective interfering particles. Curr. Top. Microbiol. Immunol. 93:151-207.

24. Pogany, J., Romero, J., Huang, Q., Sgro, J.Y., Shang, H.Z., Bujarski, J.J., 1995. De novo generation of defective interfering-like RNAs in broad bean mottle bromovirus. Virology $212,574-586$.

25. Qiu, W., Scholthof, K.B.G., 2001. Defective interfering RNAs of a satellite virus. J. Virol. 75, 5429-5432.

26. Romero, J., Huang, Q., Pogany, J., Bujarski, J.J., 1993. Characterization of defective interfering RNA components that increase symptom severity of Broad bean mottle virus infections. Virology 194, 576-584.

27. Rymelska, N., Borodynko, N., Pospieszny, H., Hasiów-Jaroszewska, B., 2013. Analysis of the biological and molecular variability of the Polish isolates of Tomato black ring virus (TBRV). Virus Genes 47, 338-346.

28. Sanfaçon, H., 2009. Nepovirus, in: van Regenmortel, M., Mahy, B. (Eds.), Desk Encyclopedia of Plant and Fungal Virology. Academic Press, pp. 235-243.

29. Steinkellner, H., Himmler, G., Sagl, R., Mattanovich, D., Katinger, H., 1992. Amino-acid sequence comparison of nepovirus coat proteins. Virus Genes 6, 197-202.

30. Szittya, G., Molnar, A., Silhavy, D., Hornyik, C., Burgyan, J., 2002. Short defective interfering RNAs of tombusviruses are not targeted but trigger post-transcriptional gene silencing against their helper virus. Plant Cell 14, 359-372. 
31. Zarzyńska-Nowak, A., Ferriol, I., Falk, B.W., Borodynko-Filas, N., Hasiów-Jaroszewska, B., 2017. Construction of Agrobacterium tumefaciens-mediated Tomato black ring virus infectious cDNA clones. Virus Res. 230, 59-62. 


\section{Figure Captions}

Fig. 1. Electrophoretic separation of TBRV RNA obtained from purified viral preparations line 1, 6 - RiboRuler High Range RNA Ladder (Thermo Scientific), line 2 - original TBRV$\mathrm{Pi}$ isolate, line 3 - TBRV-Pi after 15 passages in $C$. quinoa, line 4 - original TBRV-S1 isolate, line 5 - TBRV-S1 after 15 passages in C. quinoa.

Fig. 2. A) Structure of D RNAs derived from RNA1 of TBRV-Pi and TBRV-S1. The arrows indicate the position of primers used to amplify 5' and 3' ends of RNA1. B) Sequences near both deletion junctions of TBRV-Pi and TBRV-S1. The arrows indicate the recombinant sites and number under the arrows indicate the nucleotide adjacent to the recombination sites. The sequence marked in red of $R d R p$ appears to be an inverted repeated of 5'UTR.

Fig. 3. Plants infected with TBRV-Pi-D RNA (left column) and TBRV-Pi+D RNA (right column). A, B) C. quinoa; C, D) N. tabacum; E, F) S. lycopersicum; G, H) L. sativa.

Fig. 4. Comparison of the accumulation of the viral RNA in plants infected with TBRV-Pi+D RNA/TBRV-Pi-D RNA and TBRV-S1-D RNA/TBRV-S1+D RNA at 7, 14, 21, and 28 dpi. 
Fig 1

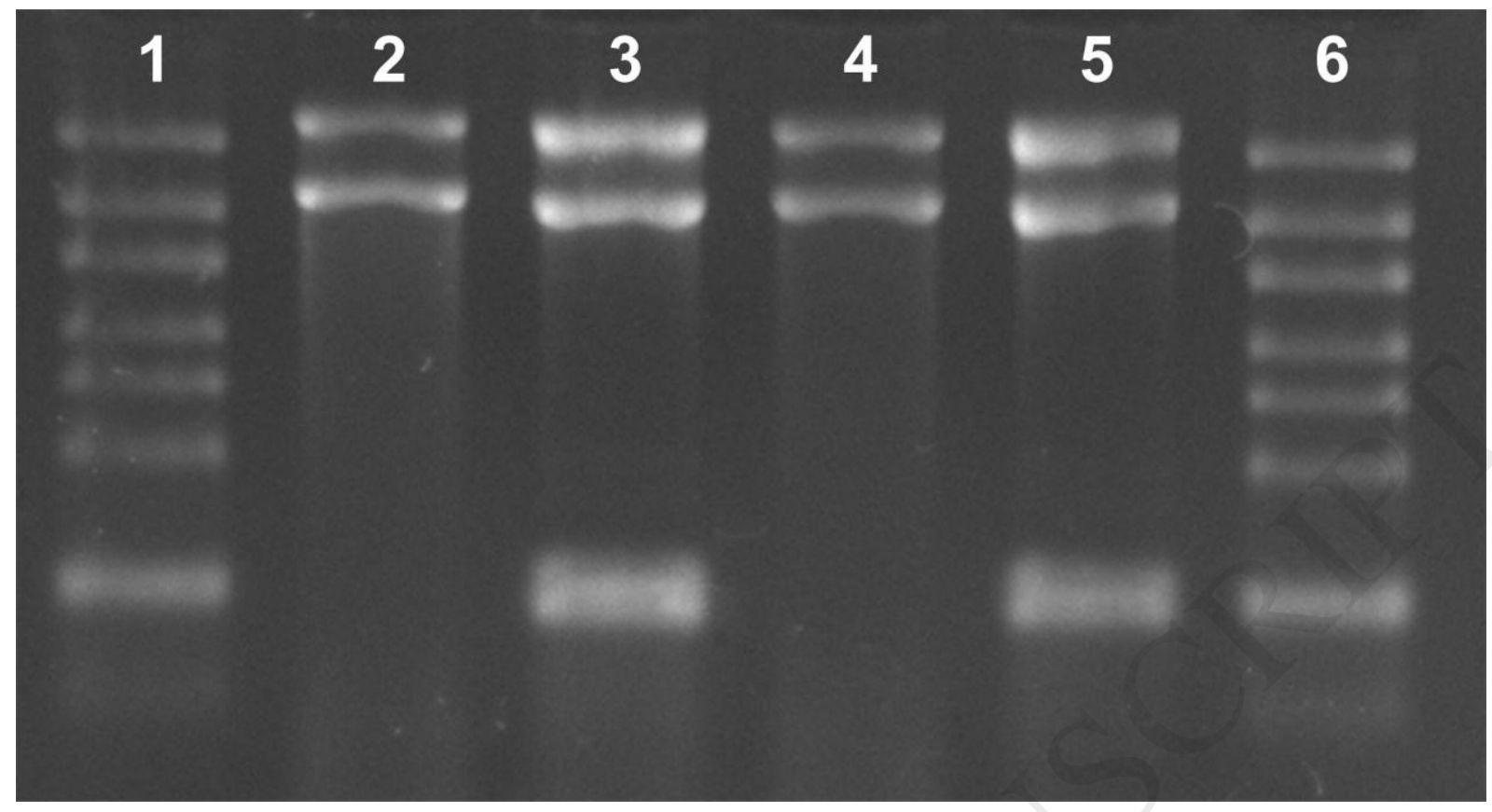


Fig 2

A
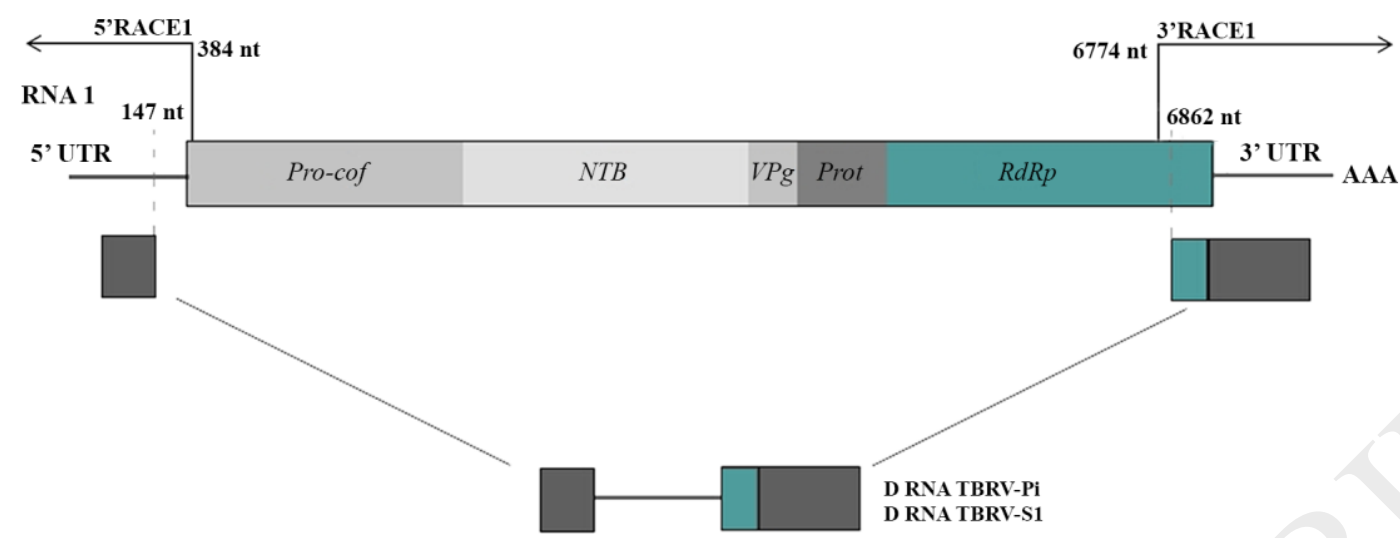

B

$110 \mathrm{nt} 147 \mathrm{nt} \quad 160 \mathrm{nt}$

5'... CUUGAUUCUGCUUUUCUUAAAUCCUGUAUCCAAUCAGG UUAUCUUUGUGGU ...

UTR

$6850 \mathrm{nt} \quad 6862 \mathrm{nt}$

UUGAAUCAGGCAG CGAAAGAUAGAAAAGUGCCAUGUAUGGUUGCACGACA9 nt

RdRp

D RNA TBRV-Pi D RNA TBRV-S1

5' UCAAAUCCUGUAACCAAUCAG \CUAAGGAA $A$ GAAAGGUACCAUGCAUGGUUGCAC .... 3' 
Fig 3

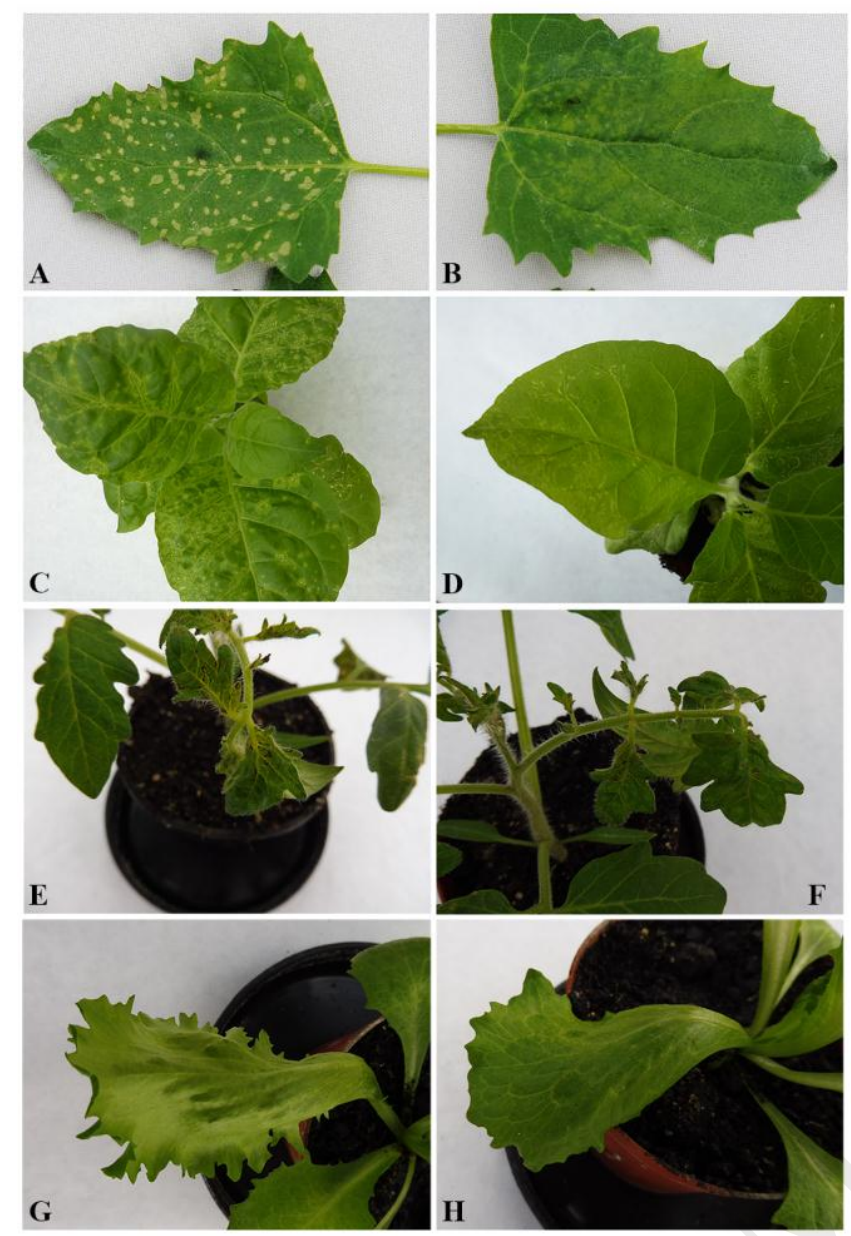


Fig 4

TBRV-Pi

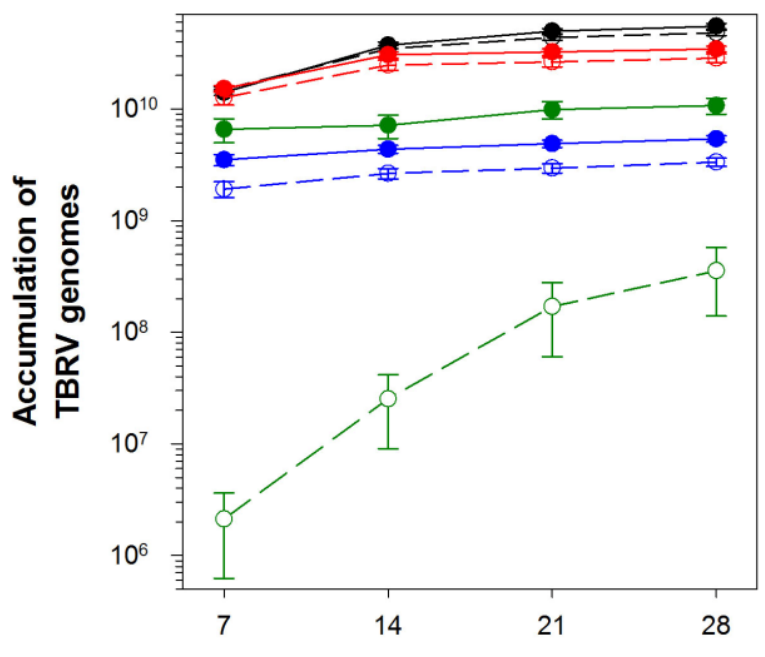

TBRV-S1

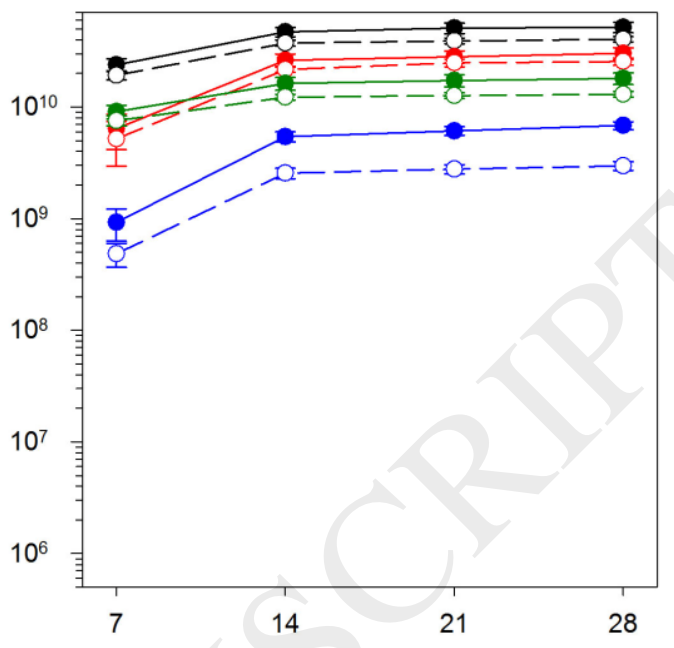

Days post-inoculation

$\longrightarrow$ C. quinoa, -D RNA

$--\mathrm{C}-\quad$ C. quinoa, +D RNA

$\longrightarrow$ N. tabacum, -D RNA

- - - N. tabacum, +D RNA

$\longrightarrow$ S. lycopersicum, -D RNA

- - - S. lycopersicum,+D RNA

$\longrightarrow$ L. sativa,-D RNA

-- - L. sativa.+D RNA 
Table 1. Results of the GLM analysis of the cumulative number of genomes. Only significant factors are included

\begin{tabular}{lrrrrrr}
\hline Source of variation & \multicolumn{1}{c}{$\boldsymbol{L} \boldsymbol{R} \boldsymbol{T}^{\mathbf{a}}$} & d.f. & \multicolumn{1}{c}{$\boldsymbol{P}$} & $\mathbf{1}-\boldsymbol{\beta}^{\mathbf{b}}$ & \multicolumn{1}{l}{$\boldsymbol{\eta}_{\boldsymbol{P}}$} & $\boldsymbol{\%}_{\text {variance }^{\mathbf{c}}}$ \\
\hline Intersection & 1450.536 & 1 & $<0.001$ & 1 & 0.896 & 0 \\
TBRV isolate $(I)$ & 8.211 & 1 & 0.004 & 0.777 & 0.013 & 3.12 \\
Presence D RNA & 86.288 & 1 & $<0.001$ & 1 & 0.126 & \\
$(D)$ & & & & & & 56.53 \\
Host species $(H)$ & 1100.280 & 3 & $<0.001$ & 1 & 0.821 & 10.16 \\
dpi $(t)$ & 386.861 & 3 & $<0.001$ & 1 & 0.454 & 3.21 \\
$I \times H$ & 89.688 & 3 & $<0.001$ & 1 & 0.131 & 0.04 \\
$D \times H$ & 11.602 & 3 & 0.009 & 0.782 & 0.018 & 0.37 \\
$D \times t$ & 10.142 & 3 & 0.017 & 0.719 & 0.016 & 10.68 \\
$H \times t$ & 267.144 & 9 & $<0.001$ & 1 & 0.341 & 0.82 \\
$I \times D \times H$ & 14.524 & 3 & 0.002 & 0.874 & 0.022 & 1.39 \\
$I \times H \times t$ & 32.392 & 9 & $<0.001$ & 0.985 & 0.049 &
\end{tabular}

${ }^{\mathrm{a}}$ Likelihood-ratio test.

${ }^{\mathrm{b}}$ Power of the test.

${ }^{\mathrm{c}}$ The fraction of observed variance explained by pure noise was $13.67 \%$. 